\title{
A Rare but Serious Complication of Ladd's Procedure: Recurrent Midgut Volvulus
}

\author{
Murat Alkan ${ }^{a}$ Pelin Oğuzkurt ${ }^{\mathrm{a}} \quad$ Ozlem Alkan $^{\mathrm{b}}$ \\ Semire Serin Ezer ${ }^{\mathrm{a}}$ Akgün Hiçsönmez ${ }^{\mathrm{a}}$ \\ Departments of a Pediatric Surgery and ${ }^{b}$ Radiology, Baskent University Faculty of \\ Medicine, Ankara, Turkey
}

\section{Key Words}

Intestinal malrotation $\cdot$ Midgut volvulus, recurrent

\begin{abstract}
An eighteen-month-old boy who had undergone a Ladd's procedure for malrotation in the newborn period presented with acute onset of nausea, vomiting, rectal bleeding, and confusion. Laparotomy revealed midgut volvulus, mesenteric lymphadenopathy and massive chylous ascites. Recurrent midgut volvulus following Ladd's procedure is extremely rare but should be borne in mind in cases of persistent or recurrent gastrointestinal symptoms. Timely surgery is necessary to avoid intestinal gangrene and decrease morbidity and mortality related to consequences of midgut volvulus.
\end{abstract}

\section{Case}

An eighteen-month-old boy was referred to the emergency department with a sudden onset of nausea and bilious vomiting which progressed into dehydration and confusion. He had low body temperature $\left(36.4^{\circ} \mathrm{C}\right)$ and blood pressure $(80 / 40 \mathrm{~mm} \mathrm{Hg})$ and increased heart rate $(160-180 / \mathrm{min})$. The past medical history revealed that he had undergone a Ladd's procedure and appendectomy for intestinal malrotation at the age of 20 days, and he had been hospitalized because of bowel obstruction which resolved with supportive treatment for three times. On admission the patient was lethargic and moderately dehydrated. A tender mid upper abdominal mass was remarkable besides the previous abdominal incision scar. There was bloody stool on rectal examination. Laboratory tests were normal. Plain abdominal radiogram showed a gasless abdomen. Ultrasonography revealed a midline abdominal mass with whirling small intestinal loops (fig. 1), massive intraperitoneal fluid and multiple enlarged mesenteric lymph nodes. After insertion of a central venous line and a nasogastric tube, broad spectrum antibiotics and a two-hour aggressive fluid therapy were initiated. The patient underwent emergency surgery. At laparotomy, there was massive chylous fluid in addition to a $540^{\circ}$ counter clock-wise rotation of the small intestine. The bowel was pale, ischemic and edematous but showed no gangrene. The appendectomized cecum was in the upper midline. The adhesions were lysed and the bowel was untwisted. The small bowel mesentery was narrow, thickened and there were multiple lymph nodes at 
the mesentery, but no lymph leakage was observed. The entire bowel was returned to the abdomen in a non-rotated position. The postoperative period was uneventful; resumption of bowel function was on the sixth postoperative day. The patient was discharged on the tenth day.

\section{Discussion}

Malrotation is a common name for a spectrum of abnormalities resulting from failure of rotation and fixation of the intestinal tract. In the most common type encountered in children, the process of rotation is incomplete and the attachment of the mesentery of the midgut to the posterior abdominal wall is narrow, so it is at risk for later twisting called volvulus. The tighter the twist, the more the midgut suffers from obstruction of the lumen, obstruction of the venous and lymphatic return and obstruction of arterial inflow, thus threatening midgut viability [1]. Most patients with malrotation develop volvulus within the first weeks of life $[2,3]$. Sudden onset of bilious vomiting in newborns or infants usually reflects duodenal obstruction or midgut volvulus due to malrotation [2]. The bowel can be strangulated at any time and age. Ischemia of the midgut results in hemodynamic instability, intractable metabolic acidosis and necrosis [1].

We present an eighteen-month-old boy who had undergone a Ladd's procedure and appendectomy for intestinal malrotation at the age of twenty days. The patient had a twoday history of bilious vomiting at that age and had undergone an elective operation. He had been hospitalized with recurrent attacks of intestinal obstruction which responded to nonoperative treatment. Before admission to our hospital intravenous fluid replacement had been initiated at the previous hospital from which the patient had been referred. Although the patient had the symptoms of dehydration, the serum electrolytes were normal. The palpable mass in the midabdomen and bloody stool in rectal examination made us think of intussusception as an initial diagnosis. Abdominal ultrasound demonstrated a whirl pool of intestines in the mid abdomen and massive abdominal fluid. These findings were suggestive of midgut volvulus. At laparotomy there was a midgut volvulus, the bowel was edematous and ischemic but no necrosis was observed. We observed that the duodeno-colic bands were incised but mesenteric base was not adequately broadened. The massive chylous ascites without a current leakage pointed out the chronicity of recurrent volvulus.

Malrotation of the intestine is usually observed in the neonatal period with signs of intestinal obstruction due to Ladd band's obstructing duodenum or midgut volvulus. Children with malrotation can present with acute or chronic symptoms or they may be completely asymptomatic. Intestinal infarction secondary to midgut volvulus is the most serious complication of this disorder. It has been emphasized that patients older than 2 years are still at risk of volvulus and rotation anomalies must be corrected surgically when they are found incidentally, even when the patients are asymptomatic $[4,5]$. Factors suggested to increase mortality are the increased percentage of necrotic bowel, the percentage of associated serious disease and younger age of the patient at operation [6].

The diagnosis is aided by the awareness of the condition and recognition of the abnormalities during radiological workup. Plain films may be normal or may show a double bubble sign, air-fluid levels or paucity of gas in the abdomen [2,3]. An upper gastrointestinal contrast study is considered the gold standard for making the diagnosis of volvulus. Small bowel is located to the right side of the abdomen with contrast narrowing at the site of obstruction causing 'corkscrewing' $[2,3]$. Barium enema may demonstrate the abnormal rotation of the cecocolic segment. Ultrasound and computed tomography may show a distended, fluid filled duodenum, increased peritoneal fluid, dilated small 
bowel loops to the right of the spine or a whirl-like pattern of bowel loops encircling the superior mesenteric artery $[2,3]$. Computed tomography as well as angiography can show the abnormal anatomy of the superior mesenteric artery [3].

Midgut volvulus is a generally acute and progressive condition which requires emergency intervention and operation. Ladd's procedure, which was described in 1932, forms the basis of the surgical treatment. The adhesions and bands between colon and duodenum and the lateral abdominal wall are divided, duodenum and small intestines are freed from rotation and left to the right of abdomen while cecum and ascending colon are placed in the left. Most authors report the permanent cure of infants and children who have undergone operations for the correction of malrotation. Although laparoscopic approach has the advantage of decreased scarring and adhesions, this technique is not adequate to examine the entire intestine.

After Ladd's procedure, bowel obstruction due to adhesion formation is a recognized risk factor. Adhesion-related obstruction is the most common and significant postoperative complication [7]. Adhesion-related obstruction is mostly happening within the first postoperative year but can occur at any time. These obstructions may respond to non-operative treatment but may require operative treatment with serious morbidity as well. While the risk of recurrent midgut volvulus is extremely low, it must be considered in patients who have undergone a Ladd's procedure formerly and have recurrent gastrointestinal symptoms. There have been discussions whether additional intestinal fixation should be carried out in patients who have undergone Ladd's procedure. It was claimed that duodenopexy and right side fixation of cecum and ascending colon would prevent a secondary volvulus. Stauffer and Herrmann [7] reported that one patient out of 28 with intestinal fixation and one out of 49 without intestinal fixation was reoperated upon for recurrent volvulus. They concluded that additional fixation after correction of malrotation is unnecessary. Mazeh et al. recommended abrasion of the parietal and visceral peritoneal surfaces when recurrence of malrotation occurs [8]. In another series reported by Feitz and Vos, of 57 patients who were operated upon for intestinal malrotation, one patient developed recurrent midgut volvulus [9]. The presence of midgut volvulus and intestinal dysmotility were suggested to be factors that cause delay of postoperative return of bowel function [9]. According to our experience, continuous gastric drip feedings with small daily increases would help the patient tolerate enteral feeding and overcome the intestinal dysmotility problem. Symptoms such as vomiting, obstipation, diarrhea, abdominal pain and cramps have been observed in those patients for more than 6 months [9]. Congenital anomalies of rotation and fixation were reported to be among the most frequent causes of chylous ascites in children [10,11]. Intermittent volvulus results in an increase of pressure and disruption of the lymphatics at the root of mesentery. The degree of lymphatic obstruction depends upon the tightness of volvulus and its chronicity [11].

\section{Conclusion}

Although the most common postoperative complication of corrected malrotation requiring surgery is bowel obstruction caused by adhesions, recurrence of the midgut volvulus should not be underestimated. Ultrasound is a non-invasive diagnostic tool for acute cases suggestive of midgut volvulus, but upper gastrointestinal barium investigation should be the conclusive diagnostic test for malrotation with or without volvulus. Despite the fact that Ladd's procedure is a satisfactory operation for correction of rotation and fixation anomalies, there is still a small but significant risk for the development of 
recurrent midgut volvulus which may end up with considerable risk of morbidity and mortality.

Fig. 1. Abdominal ultrasound shows whirling small intestinal loops (arrow) forming a midline abdominal mass.

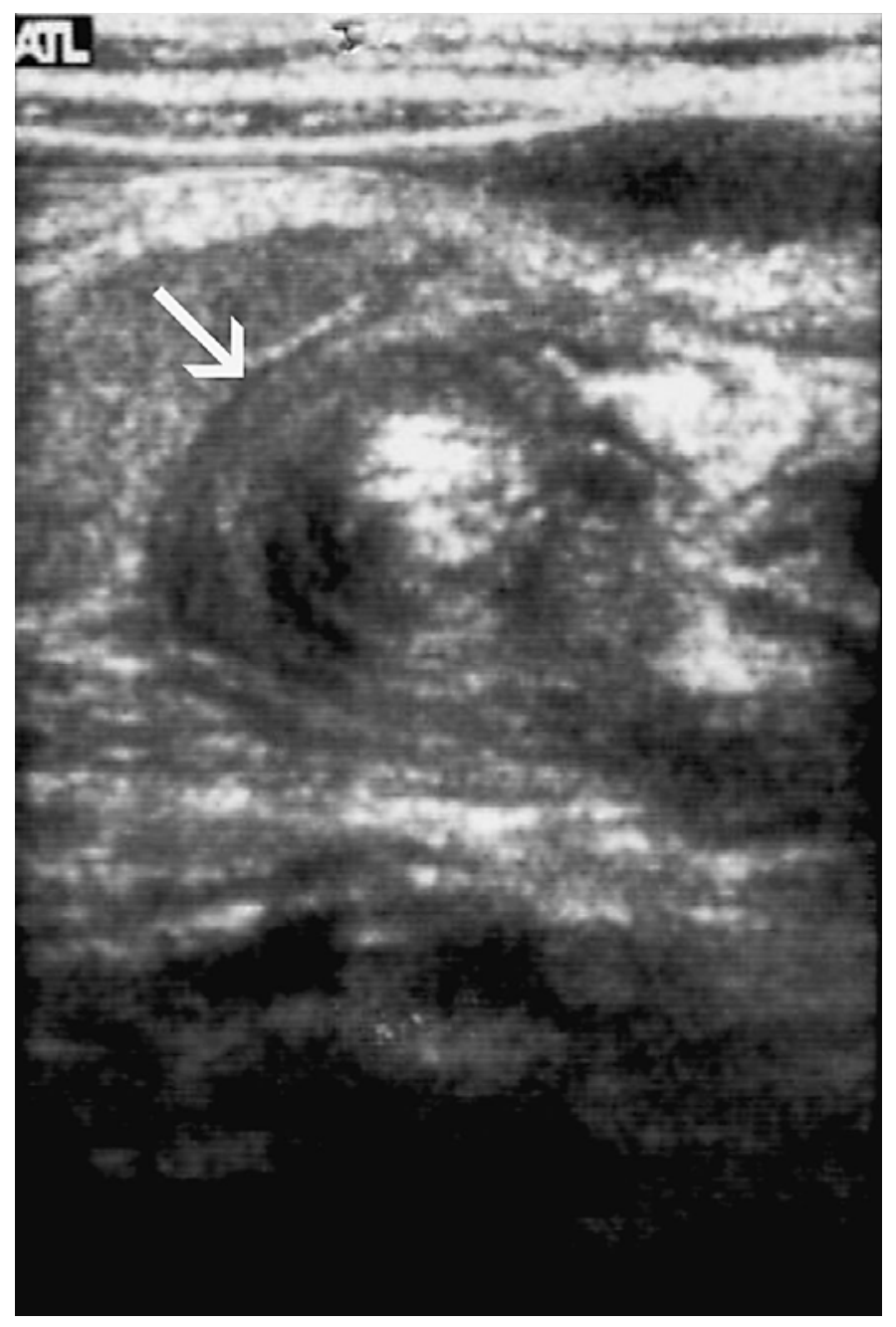




\section{References}

1 Kimura K, Loening-Baucke V: Bilious vomiting in the newborn: rapid diagnosis of intestinal obstruction. Am Fam Physician 2000;61:2791-2798.

2 McCollough M, Sharieff GQ: Abdominal surgical emergencies in infants and young children. Emerg Med Clin North Am 2003;21:909-935.

3 Rowsom JT, Sullivan SN, Girvan DP: Midgut volvulus in the adult. A complication of intestinal malrotation. J Clin Gastroenterol 1987;9:212-216.

4 Powell DM, Otherson HB, Smith CD: Malrotation of the intestines in children: the effect of age on presentation and therapy. J Pediatr Surg 1989;24:777-780.

5 Ilce Z, Celayir S, Akova F, Tekant GT, Emir H, Sarimurat N, Erdogan E, Senyuz OF, Yeker D: Intestinal rotation anomalies in childhood: review of 22 years' experience. Surg Today 2003;33:893-895.

6 Messineo A, MacMillan JH, Palder SB, Filler RM: Clinical factors affecting mortality in children with malrotation of the intestine. J Pediatr Surg 1992;27:1343-1345.

7 Stauffer UG, Herrmann P: Comparison of late results in patients with corrected intestinal malrotation with and without fixation of the mesentery. J Pediatr Surg 1980;15:9-12.

8 Mazeh H, Kaliner E, Udassin R: Three recurrent episodes of malrotation in an infant. J Pediatr Surg 2007;42:E1-E3.

9 Feitz R, Vos A: Malrotation: the postoperative period. J Pediatr Surg 1997;32:1322-1324.

10 Donnellan WL, Kimura K: Malrotation, intestinal hernias, congenital band; in Donnellan WL (ed): Abdominal Surgery of Infancy and Childhood. AustriaUnited States, Harwood Academic, 1996, pp 1-27.

11 Schwartz DL, So HB, Schneider KM, Becker JM: Recurrent chylous ascites associated with intestinal malrotation and lymphatic rupture. J Pediatr Surg 1983;18:177-179. 